\title{
Sentiment Analysis from Tweets using Convolutional Neural Networks
}

\author{
M. Pek ${ }^{1 *}$ and M. Turan ${ }^{2}$ \\ ${ }^{1}$ Istanbul Sisli Vocational School/Graphic Design, Istanbul, Turkey \\ ${ }^{2}$ Istanbul Commerce University/Computer Engineering, Istanbul, Turkey \\ *E-mail of corresponding author: mesut.pek@sisli.edu.tr
}

\begin{abstract}
By current improvements of web technology nowadays, usage of social media has increased. Twitter is a web site where millions share their opinions. Political parties, firms and other establishments has been examining data at these social media sites to learn person's opinions about themselves. Reporting the sharing of millions of persons instantly is done more easily by using machine and deep learning techniques. In this work, sentiment analysis is done by the Convolutional Neural Network which has wide-spread usage in deep learning. Besides other known works, improvements in feature selection have been applied in order to meet higher success rate. Model has been trained by the different data sets and tested in other data sets. The model has reached to $97 \%$ success rate by the training data. $90 \%$ and $89 \%$ success rates have been achieved on the tests applied to other data sets.
\end{abstract}

Received 16 February 2021

Accepted 20 April 2021

Published 03 June 2021

Keywords: Sentiment Analysis, Twitter Sentiment Analysis, Deep Learning, Convolutional Neural Network.

\section{Introduction}

User-based "social media", which emerged with the development of Web 2.0, one of the new communication technologies, offers many feedback opportunities to individual users such as instant notifications, comments, and complaints. In addition to micro blogs such as Facebook, Twitter, LinkedIn, Instagram, many platforms, including web-based news sites and even shopping sites, enables the user with instant messages and comments. In these user-based platforms that enable versatile message sharing and interactive interaction, there is a fast data flow with the messages created by the user, and these data turn into analysis objects. The platforms can analyse the analysis of the stored data on their own or through different companies.

Deep learning is a popular technique of machine learning nowadays and it is an application of artificial intelligence. Deep learning methods have been developed over the studies of artificial neural networks [1]. Deep learning includes computational models with multiple processing layers to represent the available data at multiple abstraction levels [2]. Deep learning methods, also known as deep networks, have different layers overlapping for the representation of data. Deep learning methods can create automatic feature sets by making an efficient high-level abstraction from raw data, thus enabling the automatic extraction and use of features that are usually determined by humans [1]. The learning phase of some deep learning algorithms can take quite a long

(C) The Author(s) 2021. Published by CIEES Association Press. This work is licensed under a Creative Commons Attribution-NonCommercialNoDerivs 4.0 Generic License. time, and different studies have proposed semi-supervised learning approaches to shorten the learning processes of deep neural networks [3]. In some studies, approaches to gain skills of deep learning to non-deep but widely used machine learning methods such as support vector machines have been presented [4].

Deep learning methods have yielded very successful results in processing many types of data such as video, audio, and text [5]. Often a method can be successful in text data processing such as natural language processing [6], while a different method can give more successful results in processing video and audio data. In some studies, the proposed deep learning approaches can be successfully used to learn different data types (video, audio, and text simultaneously) [7]. One of the subjects of natural language processing, which has been studied for a long time and has been an important field of application, is text classification. Deep learning is also used for text classification and there are various current studies on this subject $[8,9]$.

First deep learning using neural networks (deep learning) concept was introduced in 2000 with the work being done [10]. In [11], the authors showed that a multilayer feed forward neural network can be adjusted by a controlled back propagation method in each training it does. After this stage, various application models have been proposed. Yoon used a single layer convolution neural network in his emotional analysis (positive / negative customer reviews) on different data sets, with very successful results [8]. In another study, researchers used an iterative evolutionary neural network model for text classification. In the proposed model; iterative structure for contextual information and evolutionary neural network for text representation [9]. Hinton et al. used GPU in deep learning and implemented a 
normalization method called "dropout" to reduce memorization [11]. In another study performed with Naive Bayes, 81\% success rate was observed [12]. The success of the dropout method has been proven by the high results obtained in the ILSVRC-2012 ImageNet competition [10]. On the other hand, Ciresan et al., using $\mathrm{CNN}$, one of the deep learning methods, achieved a high success rate with only $2 \%$ error rate on the MSIT dataset [13] and reported that they found the result fastest with CNN. In another important study, a CNN-based recommendation system has been developed [14]. They analysed millions of data on the web and matched sourcetarget document pairs using vectors. Thus, they tried to group documents of interest. Although this model was not successful enough in determining the related documents, it produced good results in the subject modelling field. Akgül et al. worked on Turkish tweet messages from Twitter. They added Turkish words to the dictionary by hand. They shared that the model they developed gave successful results [15]. A Multidimensional Convolutional Neural Network (MI$\mathrm{CNN}$ ) model was proposed for two large-scale investigation of incident systems (ICEWS and GDELT) in the field of political science, a series of experiments were performed with the world protest activity data set to evaluate the model, and successful results were obtained [16]. Larsson and Nilsson created a CNN model for sentences in a field, using vectors. In their work, they applied a test optimization to resemble the word vectors of emotions. They used a Recurrent Neural Network (RNN) to decode the vector structure and for new sentences. $80 \%$ accuracy was achieved in emotion classification [17]

There are also studies using machine learning algorithms other than deep learning. Singh et al., collected on the Twitter platform tweeter Donald Trump and applied support vector machine algorithm in Weka. The algorithm was successful and predicted that Donald Trump won the results. In a different study, CNN was used to determine the gender of Russian copywriters and an accuracy of $86 \%$ was achieved [18]. Şeker and Yeşilyurt collected data from Twitter with a software called Knime, pre-processed the data set and saved it in $\mathrm{CSV}$ format. They wanted to express the emotions they guessed by processing the recorded data with emojis. In their work, they applied various machine learning algorithms in Rapid Miner data mining program. NaiveBayes algorithm gave the best result with $52 \%$ in their studies. KNN, support vector machines and decision trees algorithms could not be used due to lack of hardware [19]. Bari and Saatçioğlu used different data set of Amazon, Cornell, IMDB, Twitter, Yelp, Reviews and Kaggle in their emotion analysis studies. They conducted tests with Textblob, Opinion Finder and Stanford NLP algorithms. Textblob and Stanford had good results with NLP, but Opinion Finder did poorly [20]. Yang et al. achieved a $72.2 \%$ success rate in their study [21]. Liao et al. achieved an average success rate of $75.39 \%$ in a study they conducted using Twitter data and CNN. In the emotion analysis study performed with emojis found on Twitter, it was observed that the success rate was very low [22].
Many studies have been conducted in the literature on emotion analysis. Machine learning methods were used in the studies and the best results were used Convolution neural networks as a continuation of the study with Naive Bayes [12]. In the author's study, a maximum of $81 \%$ results were found with Naive Bayes. It is aimed to find better results with the algorithm and method we propose. In order to increase the result value, the parameters in Convolution neural networks are arranged. In the study, a $97 \%$ success rate was found by applying a different algorithm to the reference study. This result achieved $16 \%$ more success than the previous study.

\section{Method}

A string (type) is what defines or matches with a set of strings, arranged according to certain spelling rules. Regular expressions are used by many text editors, search tools, and tools for expressing specific text-based patterns. Many languages support the use of regular expressions. Regular expressions are used in data validation, data cleaning, data transformation and many other fields. Data cleansing is an important process that must be done for the model to learn well. By clearing unnecessary words and expressions in the data set, the model can be provided with a better result. With regular expressions, the URL (www, http, https), username, tag (hashtag), signs (- and /) can be cleared and the text converted to lowercase. For example: if there are tags such as@mesut in the tweet, if the model is trained without data cleaning, the model will have the possibility of misclassification and the success rate will decrease. The list of regular expressions used is shown in Table 1. The next pre-processing step is to convert the words that take the construct-inflection-possessive suffixes into a single form. For this study, which is of great importance in frequency calculation, stemming algorithms are used. For this purpose, PorterStemmer root finding algorithm, which is the most widely known of the algorithms developed for the English language, was applied.

Table 1. List of regular expressions.

\begin{tabular}{c}
\hline Regular expressions \\
\hline Replace URL \\
Replace Username \\
Replace Hashtag \\
Replace With Space \\
\hline
\end{tabular}

The structure diagram used when making emotion analysis from tweets with machine learning is shown in Figure 1 and the system model suggested in the intermediate stages is shown in Figure 2.

The words in the data set are first passed through the data cleaning stage. It includes data cleaning, parsing URL, name, tag, and converting all to lowercase. With the conversion to lowercase, the words that are separated by normalization are ensured to be calculated accurately. If the data cleaning step is not implemented, the success rate of the system decreases, and runtime increases because the model cannot be properly trained. The success rate increases when you remove ineffective words and names. 


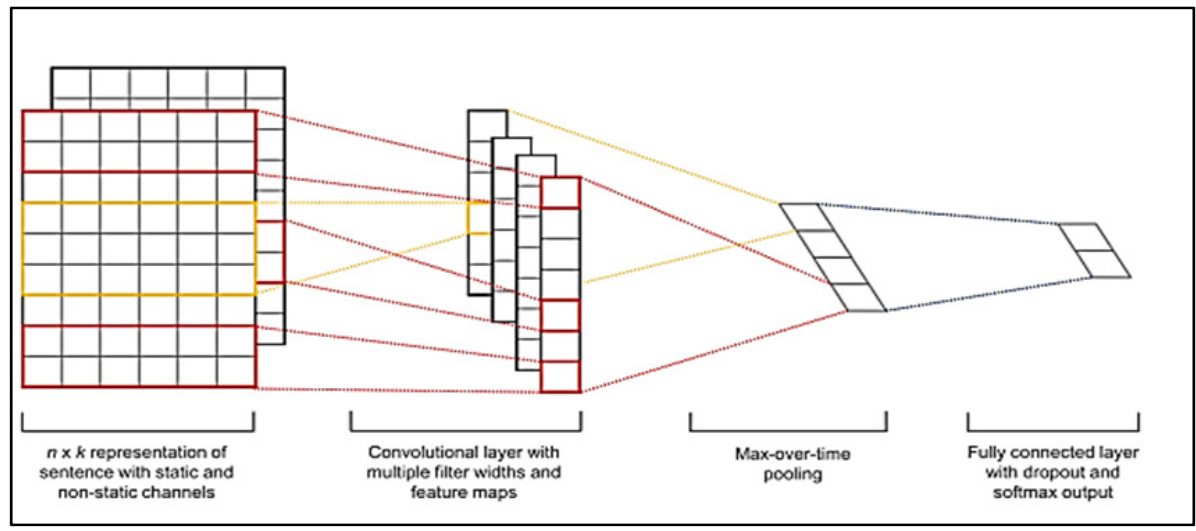

Fig. 1. CNN Model Used.

We tried many parameters for the model to find the best values and CNN parameters, for which we reached the best success rate experimentally, are given in Table 2 . After the model is trained, tests can be performed according to any data set.

Table 2. CNN Parameters.

\begin{tabular}{cc}
\hline Parametre Adı & Parametre Değeri \\
\hline embedding_dim & 128 \\
filter_sizes & 3 \\
num_filters & 128 \\
batch_size & 64 \\
num_epochs & 200 \\
fc_hidden_size & 1024 \\
\hline
\end{tabular}

\section{Data sets}

Kaggle is a platform where data sets used in machine learning are available and competitions are held. Both data sets used in the study were taken from this site. There are two main pieces of information that we will use in the data set: emotion and tweet content. The first data set is used to train our model and the second data set is used for testing. The data set used for educational purposes contains 100.000 tweets.

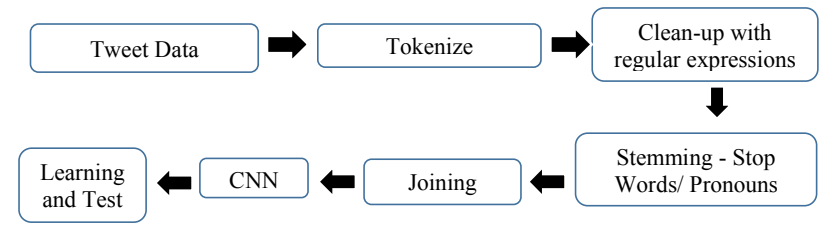

Fig. 2. Proposed system model.

The structure and sample data of the data set we used when training the system for the first time are shown in Figure 3.

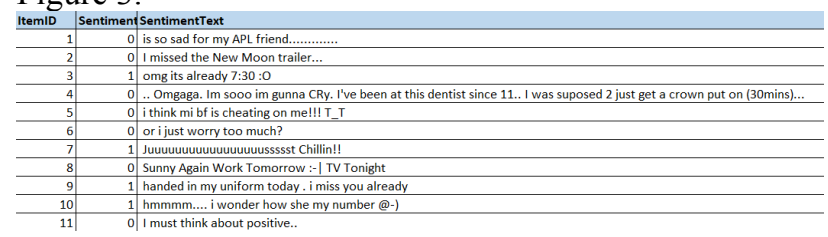

Fig. 3. Learning data set.
The structure and sample data of the data set we use to test the model are shown in Figure 4 and Figure 5.

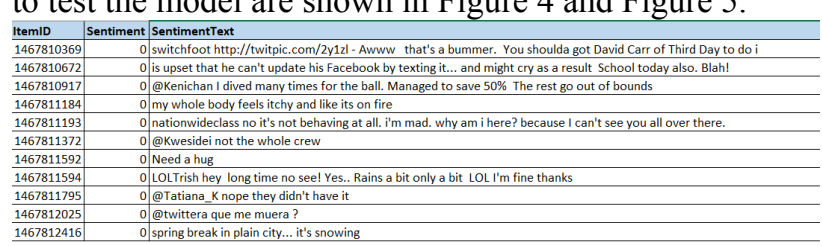

Fig. 4. Test data set.

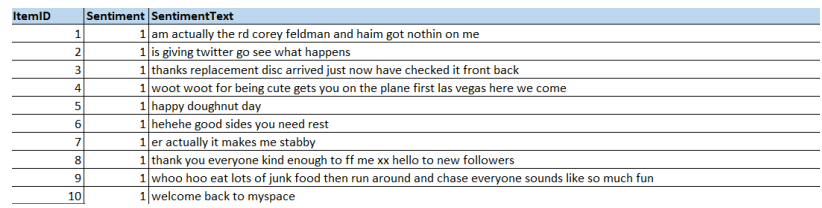

Fig. 5. Test data set.

The structure and sample data of the data set we use to test the model are shown in Figure 4 and Figure 5.

While the model is being trained, stopwords and nouns are removed from sentences to increase the success rate.

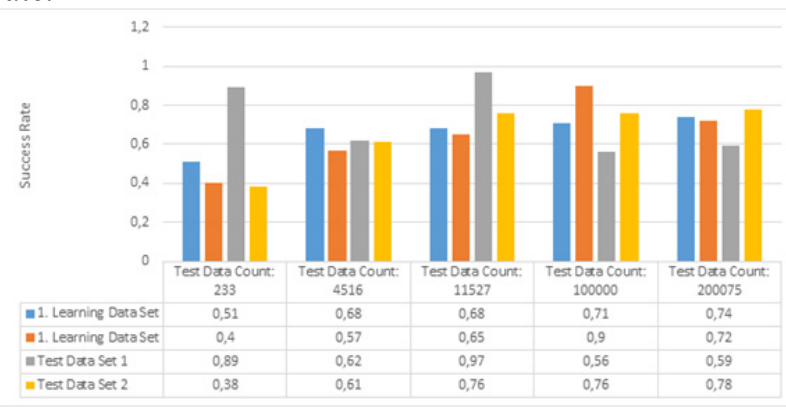

Fig. 6. Learning rate while training model.

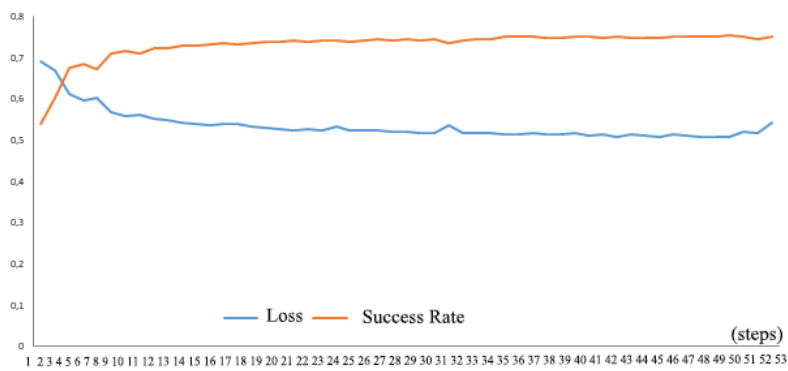

Fig. 7. Success rate of test using the trained model. 
As can be seen in Figure 6, in the tests performed while training the model, the success rate was low, and the loss value was at the maximum level in the first training phase.

It was observed that when the training time of the model increased, the success rate increased, and the loss value decreased. A 97\% success rate was observed in the tests performed during the training by downloading a different data set from the Kaggle site.

An example of the tests performed can be seen in Figure 7 and the graph in Figure 6 had the highest success rate of $97 \%$. In [23], 71\% success rate was observed in the first stage and then $72 \%$.

\section{Conclusion}

While training the model, the number of layers, hidden layers and linked layers are arranged for performance and hardware upgrades have been made to increase the performance on the computer. In the training, the model was trained using the first data set. In the trained model, $74 \%$ success rate was achieved in the tests performed with the first data set and data not used in learning. When the training time was reduced, it was seen that the success rate of $90 \%$ was obtained in the tests performed with the 1 st data set. In the tests, different data sets and our $\mathrm{CNN}$ model were tested and the success rate in the first data set we used gave the best result. When the success rates in our study were examined, it was seen that Salur's work was successful as a success rate [23]. According to a study in the literature, it has been observed that our study with convolutional neural networks gave the best result [24]. When the success rate is examined, success rates close to $75 \%$ have been shared in the studies in the literature. In our study, a success rate of \% was observed in studies performed with different tweet data sets.

\section{References}

[1] Ravì, D., Wong, C., Deligianni, F., Berthelot, M., Andreu-Perez, J., Lo, B., \& Yang, G. Z. (2007), Deep learning for health informatics, IEEE Journal of Biomedical and Health Informatics, vol. 21, no. 1, 4-21, doi: 10.1109/JBHI.2016.2636665

[2] Bengio, Y. (2009). Learning Deep Architectures for AI. Found. Trends Mach. Learn. Vol. 2, no. 1, 1-127, https://doi.org/10.1561/2200000006

[3] Lee, D. H. (2013). Pseudo-label: The simple and efficient semi-supervised learning method for deep neural networks. CML 2013 Workshop: Challenges in Representation Learning (WREPL), Atlanta, Georgia, USA, 1-6.

[4] Cho, Y. \& Saul, L. K. (2009). Kernel methods for deep learning. 22nd International Conference on Neural Information Processing Systems (NIPS'09). Red Hook, NY, USA, 342-350.

[5] LeCun, Y., Bengio, Y. \& Hinton, G. (2015). Deep learning. Nature vol. 521, 436-444, https://doi.org/10.1038/nature14539

[6] Collobert R. \& Weston, J. (2008) A unified architecture for natural language processing: Deep neural networks with multitask learning", 25th international conference on Machine learning (ICML '08), Helsinki, Finland, 160-167, https://doi.org/10.1145/1390156.1390177

[7] Ngiam, J., Khosla, A., Kim, M., Nam, J., Lee, H. \& Ng, A. Y. (2011). Multimodal deep learning, 28th International Conference on Machine Learning, Washington, USA, 689-696.
[8] Kim, Y. (2014). Convolutional neural networks for sentence classification. Proceedings of the 2014 Conference on Empirical Methods in Natural Language Processing. DOI: 10.3115/v1/D14-1181

[9] Lai, S., Xu, L., Liu, K., Zhao, J. (2015). Recurrent convolutional neural networks for text classification. Proceedings of the Twenty-Ninth AAAI Conference on Artificial Intelligence (AAAI'15). AAAI Press, 2267-2273.

[10] Hinton, G.E., Srivastava, N., Krizhevsky, A., Sutskever, I., \& Salakhutdinov, R. (2012). Improving neural networks by preventing co-adaptation of feature detectors. ArXiv, abs/1207.0580.

[11] Aizenberg I., Aizenberg N. \& Vandewalle J. (2000). Multi-Valued and Universal Binary Neurons: Theory, Learning and Applications. Springer-Verlag, https://doi.org/10.1007/9781-4757-3115-6.

[12] Pek M. \& Turan M. (2019). Sentiment Analysis of Tweets Using Machine Learning, International Conference on Data Science, machine Learning and Statistics, Turkey, Van, 85-87.

[13] Ciresan D., Meier U. \& Schmidhuber J. (2011). Flexible, High Performance Convolutional Neural Networks for Image Classification. International Joint Conference on Artificial Intelligence IJCAI-2011. pp. 1237-1242. DOI: 10.5591/978-157735-516-8/IJCAI11-210.

[14] Gao J., Deng L., Gamon M. \& He X. (2014). Modeling interestingness with deep neural networks. Proceedings of the 2014 Conference on Empirical Methods in Natural Language Processing (EMNLP), Doha, Qatar, 2-13, https://doi.org/10.3115/v1/D14-1002

[15] Akgül E. S., Ertano C. \& Diri B. (2016). Sentiment analysis with Twitter. Pamukkale University Journal of Engineering Sciences, vol. 22 no. 2, 106-110. (in turkish).

[16] Chen T., Xu R., He Y., \& Wang X. (2016). Improving sentiment analysis via sentence type classification using BiLSTM-CRF and CNN. Expert Systems with Applications, Elsevier, Vol. $\quad 72, \quad$ 221-230, https://doi.org/10.1016/j.eswa.2016.10.065

[17] Larsson, M., \& Nilsson, A. (2017). Manifold Traversal for Reversing the Sentiment of Text.

[18] Singh P., Sawhney R.S. \& Kahlon K.S. (2017). Forecasting the 2016 US Presidential Elections Using Sentiment Analysis. In: Kar A. et al. (eds) Digital Nations - Smart Cities, Innovation, and Sustainability. I3E 2017. Lecture Notes in Computer Science, vol. 10595. Springer, Cham. https://doi.org/10.1007/978-3-319-68557-1 36

[19] Şeker, Ş. E., Yeşilyurt A. (2017) Twitter Sentiment Analysis using Text Mining Methods. YBS Encyclopedia, vol. no.2, 26-36. (in turkish).

[20] Bari A. \& Saatçioğlu G., (2018). Emotion Artificial Intelligence Derived from Ensemble Learning, 17th IEEE International Conference On Trust, Security And Privacy In Computing And Communications/ 12th IEEE International Conference On Big Data Science And Engineering (TrustCom/BigDataSE), 1763-1770, doi: 10.1109/TrustCom/BigDataSE.2018.00266

[21] Yang X, Macdonald C., Ounis L. (2018). Using word embeddings in Twitter election classification. Inf Retrieval $J$, vol. 21, pp. 183-207. https://doi.org/10.1007/s10791-017-93195

[22] Liao S., Wang J., Yu R., Sato K. \& Cheng Z. (2017). CNN for situations understanding based on sentiment analysis of twitter data. Procedia Computer Science, Vol. 111, 376-381, https://doi.org/10.1016/j.procs.2017.06.037

[23] Salur M. U. \& Aydin İ. (2018). The Impact of Preprocessing on Classification Performance in Convolutional Neural Networks for Turkish Text. International Conference on Artificial Intelligence and Data Processing (IDAP), Malatya, Turkey, 1-4, doi: 10.1109/IDAP.2018.8620722. 
[24] Zhao, L., \& Zeng, C. (2017). Using Neural Networks to Predict Emoji Usage from Twitter Data.

[25] Tantuğ, A. C. (2012). Metin Sinıflandırma, Türkiye Bilișim Vakfi Bilgisayar Bilimleri ve Mühendisliği Dergisi.

[26] Şeker, A., Diri, B. \& Balik, H. (2017). Derin Öğrenme Yöntemleri ve Uygulamaları Hakkında Bir İnceleme, Gazi Mühendislik Bilimleri Dergisi.

[27] Salakhutdinov, R., Mnih, A. \& Hinton, G. (2007). Restricted Boltzmann machines for collaborative filtering, in
Proceedings of the 24th international conference on Machine learning - ICML.

[28] Pek, M. \& Turan, M. (2019). Evrişimli Sinir Ağlari Kullanarak Imdb Film Yorumlarindan Duygu Analizi, Akademisyen Kitabevi A.Ş.

[29] Pek, M. (2019). Ağlarda Saldiri Tespit Ve Önleme Sistemleri, Akademisyen Kitabevi A.Ş.

[30] Boynukalın, Z. (2012). Emotion Analysis of Turkish Texts by Using Machine Learning Methods, MSc Thesis, Middle East Technical University. 